\title{
Solar $\boldsymbol{p}$-modes and surface magnetic fields: Is there an acoustic emission?
}

\section{MDI/SOHO observations}

\author{
R. Jain ${ }^{1}$ and D. Haber ${ }^{2}$ \\ 1 Department of Physics, UMIST, Manchester M60 1QD, UK \\ e-mail: rjain@suraj.phy.umist.ac.uk \\ 2 JILA, University of Colorado, Boulder, CO 80309-440, USA \\ e-mail: dhaber@solarz.colorado.edu
}

Received 18 July 2001 / Accepted 28 February 2002

\begin{abstract}
We study the effect of magnetism on the surface amplitude of $p$-modes by creating power maps using Doppler velocity, line-depth and continuum intensity data taken by the Michelson Doppler Interferometer (MDI) instrument on board SOHO. This analysis, using proper tracking procedures, of MDI line-depth data and its comparison with the simultaneous Doppler velocity data is the first such analysis. All three datasets show that the $p$-mode power is suppressed in the magnetic region with suppression increasing with field strength. However, in the high-frequency range, the power is enhanced in the Doppler velocity and line-depth data but not in continuum intensity. This enhancement, when present, appears to be in intermediate field strength elements in the immediate vicinity of a magnetically active region.
\end{abstract}

Key words. Sun: helioseismology, oscillations, magnetic fields, photosphere, sunspots - methods: data analysis

\section{Introduction}

One of the most interesting studies in helioseismology is the effect of magnetic fields on the frequencies and the surface amplitudes of $p$-modes. Such studies are important not only for understanding the nature of the propagation of waves in the solar atmosphere but also for providing information about the surface and the cavity within which these modes propagate. Also, such studies can be useful as a seismic probe of magnetically active regions. It has been known that the surface amplitude of $p$-modes are suppressed in magnetically active regions (Woods \& Cram 1981; Lites, et al. 1982; Tarbell et al. 1988; Brown et al. 1992; Brown 1994). Many possible mechanisms have been suggested to explain such observed suppression (Cally 1995; Jain et al. 1996; Hindman et al. 1997; see also Goldreich \& Keely 1977; Goldreich \& Kumar 1988). On the other hand, the power measured using various spectral lines suggest that it is enhanced around magnetic field regions at frequencies above $5 \mathrm{mHz}$ (Braun et al. 1992; Brown et al. 1992; Brown 1994; Jain \& Haber 1997; Hindman \& Brown 1998; Thomas \& Stanchfield 2000).

Send offprint requests to: R. Jain,

e-mail: rjain@suraj ·phy . umist.ac.uk
These ring-like regions of enhanced power surrounding magnetically active regions such as pores or sunspots are sometimes referred to as a "halo" of enhanced power and are believed to be due to increased acoustic emission (Braun et al. 1992; Brown et al. 1992). However, the absence of these halos in the MDI continuum intensity data led Hindman \& Brown (1998) to suggest that the halos seen in the Doppler velocity data could be due to incompressible waves.

In this paper we investigate the frequency dependence of suppression and enhancement of acoustic power over magnetic field regions for each of the datasets-Doppler velocity, line-depth and continuum intensity. We also study how this power varies as a function of the magnetic field for particular frequency ranges. These datasets were taken by the Michelson Doppler Interferometer (MDI) instrument on board SOHO.

The analysis of MDI line-depth data and its comparison with the simultaneous Doppler velocity data is the first such analysis. For the first time, the same analysis has been carried out on two different datasets, one containing a sunspot and the other a plage. In addition, this work has made available the comparison of full disk data with high resolution data. The present study clearly suggests 
that the same physical mechanisms which cause power suppression and enhancement are responsible in sunspot and plage regions.

The findings reported in this paper are based on the analysis carried out on a number of datasets. In this paper, we present a few representative examples. The paper is organized as follows. In Sect. 2, we briefly describe the Michelson Doppler Interferometer (MDI) data and the analysis procedure. Section 3 deals with various results regarding the power suppression/enhancement and compares magnetic and non-magnetic regions. In Sect. 4, we discuss these results and their implications.

\section{Data analysis}

We consider two datasets - 1996, June 6 full disk $\left(2^{\prime \prime} /\right.$ pixel $)$ data and 1997, January 17, high resolution $\left(0.6^{\prime \prime} /\right.$ pixel $)$ data. Table 1 describes the details of these datasets.

For the first dataset, the observations were made during the Dynamics Campaign of the MDI instrument aboard SOHO on June 6, 1996. We therefore have 1536 min (25.6 hours) of both Doppler velocity data and line-depth data that were taken nearly simultaneously (see Scherrer et al. 1995 for details on the instrument and how the observables are formed) with a one-minute cadence and a $99 \%$ duty cycle. From the $1024 \times 1024$ pixel calibrated images, three regions of $256 \times 256$ pixels (each) were tracked and remapped using Postel's projection (preserves great circles; see Bogart et al. 1995). The first region was near disk center over a sunspot group, the second was over a decaying active region, and the third was over quiet (weakly magnetised) sun. Magnetograms, which were taken every $96 \mathrm{~min}$ with the same instrument, were also tracked and remapped in the same manner as the other data. These magnetograms are used to provide the magnetic field strengths in the later analysis. Figure 1 (top panel) shows representative tracked and remapped magnetograms of the sunspots and active region. The magnetic field within the pair of sunspots is fairly weak (maximum $B+850 \mathrm{G}$ and $-1.2 \mathrm{kG}$ ).

For the high resolution data containing a Plage, we have 240 min of Doppler velocity data and continuum intensity data. The region was tracked in order to keep the Plage at the same location. The disk center at the middle of the run was at (-4.9 deg, $198 \mathrm{deg}$ ); thus (cf. Table 1), the Plage region is very close to the disk center.

To provide the best comparison between the two data sets, a four hour subset of the 25.6 hour data is used. It has been found that the result is not critically sensitive to whether the subset is taken at the beginning of the 25.6 hour period or at some point later on. Although some changes in the magnetic field are seen during these four hours; they are not very significant and we average magnetograms. The positive polarity is represented by bright colour whereas the dark colour represents the negative polarity in the averaged magnetograms.
Sunspots
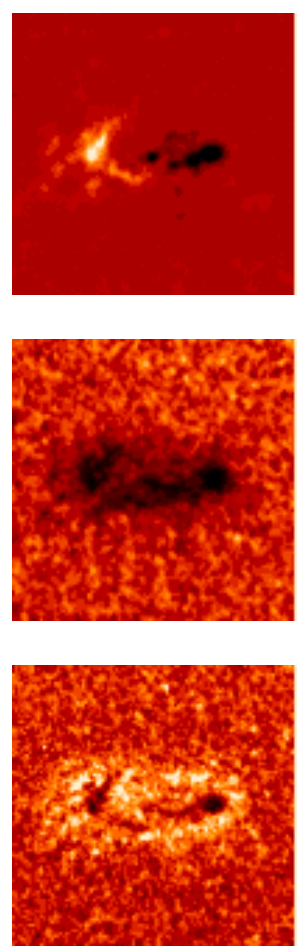

Fig. 1. The top panel (A and B) shows the tracked and remapped magnetograms from 6 June 1996. The sunspot data is centered at heliographic coordinates (3.04 ${ }^{\circ}$ lat., $291^{\circ}$ long.) and the active region at $\left(-4.96^{\circ}\right.$ lat., $269^{\circ}$ long. $)$. The middle and bottom panel show doppler power images constructed by integrating over frequency ranges $2 \leq \nu \leq 4 \mathrm{mHz}$ ( $p$-band; C for sunspots and $\mathrm{D}$ for active region) and $5.5 \leq \nu \leq 7.5 \mathrm{mHz}$ (high- $\nu$ band; E for sunspots and $\mathrm{F}$ for active region) respectively. Brighter pixels represent higher power. Note that the $p$-band power is suppressed in regions of strong magnetic fields, with the most suppression in regions of strongest magnetic field, whereas the high- $\nu$ band shows power enhancement in regions of reasonably strong magnetic field but still shows suppression in regions of strongest field.

The above mentioned Doppler velocity, line depth and continuum intensity datasets are Fourier transformed in time at each pixel to form power maps. The effects of granulation are reduced by filtering out signals below the $f$-mode frequencies in the frequency-wavenumber domain.

\section{Results}

Figure 1 (middle and bottom panels) shows summed images of the Doppler power contained within the frequency ranges $2 \leq \nu \leq 4 \mathrm{mHz}$ ( $p$-band) and $5.5 \leq \nu \leq 7.5 \mathrm{mHz}$ (high- $\nu$ band) of the 1996, June 6 data, while Fig. 2 shows the line-depth data treated similarly. The darker and brighter pixels represent lower and higher power respectively. It is worth mentioning that the results remain unchanged when frequency ranges $2 \leq \nu \leq 5 \mathrm{mHz}$ and $5.5 \leq \nu \leq 6.5 \mathrm{mHz}$ were used for the $p$-band and high$\nu$ band respectively. 
Table 1. Different datasets used for analysis.

\begin{tabular}{lll}
\hline \hline & 1996 June 6-7 & 1997 Jan. 17 \\
\hline Regions & Sunspots, Active \& Quiet & Plage \\
Type of data & Doppler vel. \& line depth & Doppler vel. \& cont. intensity \\
Start \& End times (TAI) & $09: 56: 30 \& 11: 31: 30$ & $20: 00 \& 23: 59$ \\
Duration (hours) & 25.6 & 4 \\
Field of view & $512^{\prime \prime} \times 512^{\prime \prime}$ & $270^{\prime \prime} \times 270^{\prime \prime}$ \\
Center location (region) & $\left(3.04^{\circ}, 291^{\circ}\right)$ & $\left(-4.6^{\circ}, 200^{\circ}\right)$ \\
Resolution (per pixel) & $2^{\prime \prime}$ & $0.6^{\prime \prime}$ \\
Cadence (s) & 60 & 60 \\
Nyquist freq. $(\mathrm{mHz})$ & 8.333 & 8.333 \\
Freq. spacing, $\Delta \nu(\mu \mathrm{Hz})$ & 101.85 & 69.44 \\
\hline
\end{tabular}

Sunspots
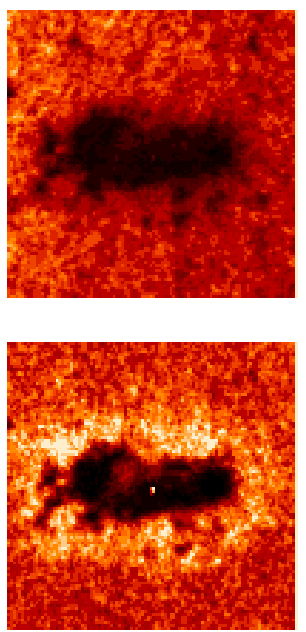

Active Region
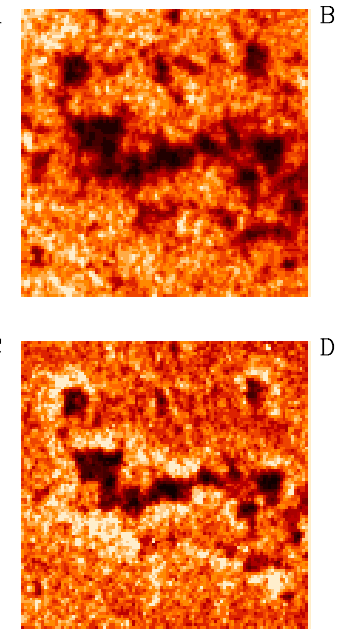

$5.5-7.5 \mathrm{mHz}$
$2.0-4.0 \mathrm{mHz}$

Fig. 2. As in Fig. 1 except that here line-depth intensity data is used for constructing images of power. Here, $p$-band (A and $\mathrm{B})$ and high-frequency band ( $\mathrm{C}$ and $\mathrm{D})$ show power suppression in regions of strong magnetic fields but high frequencies also show power enhancement around such regions when the field strength isn't too high.

Let us first consider the Doppler velocity data (Fig. 1; middle and bottom panels). The comparison of the Doppler power in the magnetic and the surrounding nonmagnetic regions suggests that the power is suppressed in the magnetic region within the $p$-band and enhanced in such regions in the high- $\nu$ band although in the case of the sunspot there is still some suppression for very strong magnetic field (see the images labelled as A and E). No significant net power suppression or enhancement is found in the quiet sun region (not shown here). Note that the power map shows small scale variations even in the non-magnetic region. We believe that these are due to sources and sinks of $p$-mode emission (see Haber et al. 1999). Figure 2 shows a pattern similar in general but not in the specifics. In the line-depth data the power is suppressed in a magnetic region in both the $p$-band and the high- $\nu$ band. In the high- $\nu$ band it is enhanced further out. We repeat this analysis on the high resolution data to see if the finer grid-scale changes our results.

Figure 3 shows the Doppler power images (labelled A and D) and continuum intensity power images (labelled B and $\mathrm{E}$ ) for the 1997 January 17 plage region. The images in the top and bottom panels are constructed by summing power over frequency ranges $2 \leq \nu \leq 4 \mathrm{mHz}$ ( $p$-band) and $5.5 \leq \nu \leq 7.5 \mathrm{mHz}$ (high- $\nu$ band) respectively. We also show an averaged magnetogram (middle panel) of the same region for comparison. Bright and dark colour represent positive and negative polarity respectively in the magnetograms. Note that the $p$-band power is suppressed in the regions of strong magnetic field. In the high- $\nu$ band the Doppler power (labelled D) shows enhancement around the regions of strong fields whereas the continuum intensity (labelled E) still shows suppression. A careful comparison between the Doppler velocity map "D" and the magnetogram "C" shows that there is still power suppression in the elements with very strong field. Note that the intensity data shows a better correlation with the magnetic fields (compare "E" and "C").

Figures 1-3 clearly suggest that there is a link between magnetic fields, $p$-mode power and the frequencies of $p$-modes. We make an attempt to quantify this link. In what follows, we study the variation of power as a function of frequency and magnetic field strength. Recall that the magnetograms measure the line of sight component. It is worth mentioning that the MDI high-resolution data have an uncertainity of about $\pm 20 \mathrm{G}$ in their measurements. Thus, the pixels with $0 \mathrm{G}$ and weak magnetic field strength are important. They may also mean a predominantly perpendicular field in those pixels. Also, the MDI magnetic field measurements saturate at low values so the maximum field strengths $(+850 \mathrm{G}$ and $-1.2 \mathrm{kG}$ for June 6 , 1996 and $+695 \mathrm{G}$ and $-653 \mathrm{G}$ for the plage region) are not realistic.

A careful investigation of the datasets suggests two important things: (1) Both the largest power suppression in $p$-band and the largest power suppression in the high- $\nu$ band coincides with the highest magnetic field strength. (2) The average $p$-mode power in the non-magnetic (or 
Velocity

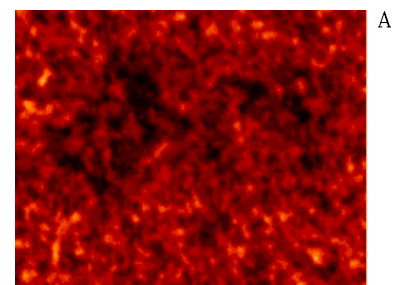

Intensity

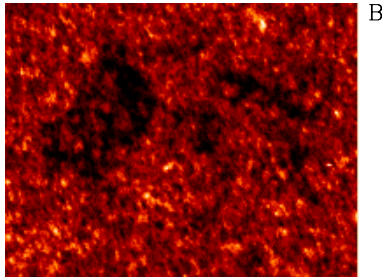

$2.0-4.0 \mathrm{mHz}$

Magnetogram
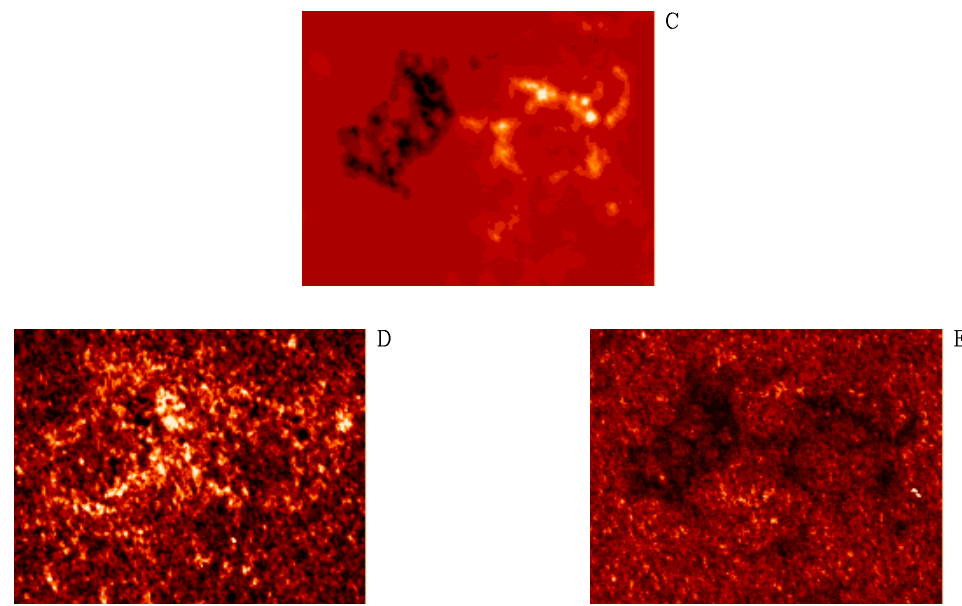

$5.5-7.5 \mathrm{mHz}$

Fig. 3. The top panel shows the power images (Doppler velocity (A) and continuum intensity image (B)) constructed by summing over frequency ranges $2 \leq \nu \leq 4 \mathrm{mHz}$ for 1997 January 17, high resolution plage region. The middle panel (C) shows the averaged magnetogram of the Plage region to facilitate comparison. The bottom panel shows the power images (Doppler velocity (D) and continuum intensity image (E)) in the frequency range $5.5 \leq \nu \leq 7.5 \mathrm{mHz}$. Note that $p$-band power (A and B) is suppressed in the regions of strong magnetic field whereas, the Doppler power in the high- $\nu$ band (D) is enhanced in the same regions.

weakly magnetic) pixels near the sunspots or plage is less than the average $p$-mode power in such pixels further away from these regions (where there is no significant level of magnetic activity). Thus, instead of using any particular normalisation procedure (which can bias the quantitative results), we prefer to show power and the corresponding magnetic field strength at each pixel for the two frequency bands. In Fig. 4, we plot the power as a function of magnetic field strength for the continuum intensity and Doppler velocity data of January 17, 1997 Plage region. The top and bottom panels show the plots for the $p$-band and the high- $\nu$ band respectively. Note that for the velocity and the intensity data, the $p$-band power reduces by a factor of greater than 2 in going from field-free regions to regions with magnetic fields $|B| \geq 400 \mathrm{G}$. It is also clear from Fig. 4, that the intensity power is suppressed in strong magnetic field regions in both frequency bands and that there is more suppression for stronger fields. It appears that the slight enhancement of Doppler power that we see in high- $\nu$ band (see Fig. 4) is coming from the pixels with intermediate field strengths $(50 \leq|B|<300 \mathrm{G})$. In order to highlight these trends, we show in Fig. 5, the Doppler power (for June 6,1996 case) as a function of frequency for two ranges of magnetic field strengths $0 \leq|B|<50 \mathrm{G}$ (shown with dashed line) and $51<|B|<300 \mathrm{G}$ (shown with solid line).
It clearly indicates that the power is suppressed in the $p$-band for the intermediate field strengths but enhanced above $5.2 \mathrm{mHz}$. A similar conclusion can be drawn for the Doppler velocity data of the plage region (see Fig. 6 curves a and b). Figure 6 (curves $c$ and d shows the suppression of continuum intensity power for all frequencies for the same two ranges of magnetic field strengths. We also find:

1) For $|B| \geq 100 \mathrm{G}$, the power is suppressed more for line-depth data than for the Doppler velocity data in $p$-band $(2-4 \mathrm{mHz})$;

2) In high- $\nu$ band $(5.5-7.5 \mathrm{mHz})$, the Doppler velocity data show power enhancement around strong mag. field regions in the range $50 \leq|B| \leq 300 \mathrm{G}$ whereas the line-depth data show the same for weaker fields. The enhancement seen in high- $\nu$ band in the line depth data (see Fig. 2) corresponds to weak magnetic field pixels $5 \leq|B|<20 \mathrm{G}$ around the sunspots.

In general, the power suppression in the $p$-band is highest at about $3 \mathrm{mHz}$ and the power enhancement in the high- $\nu$ band is largest at about $6 \mathrm{mHz}$.

\section{Discussion}

There is suppression of power in the $p$-band in magnetic regions for both velocity and intensity data. If we assume 

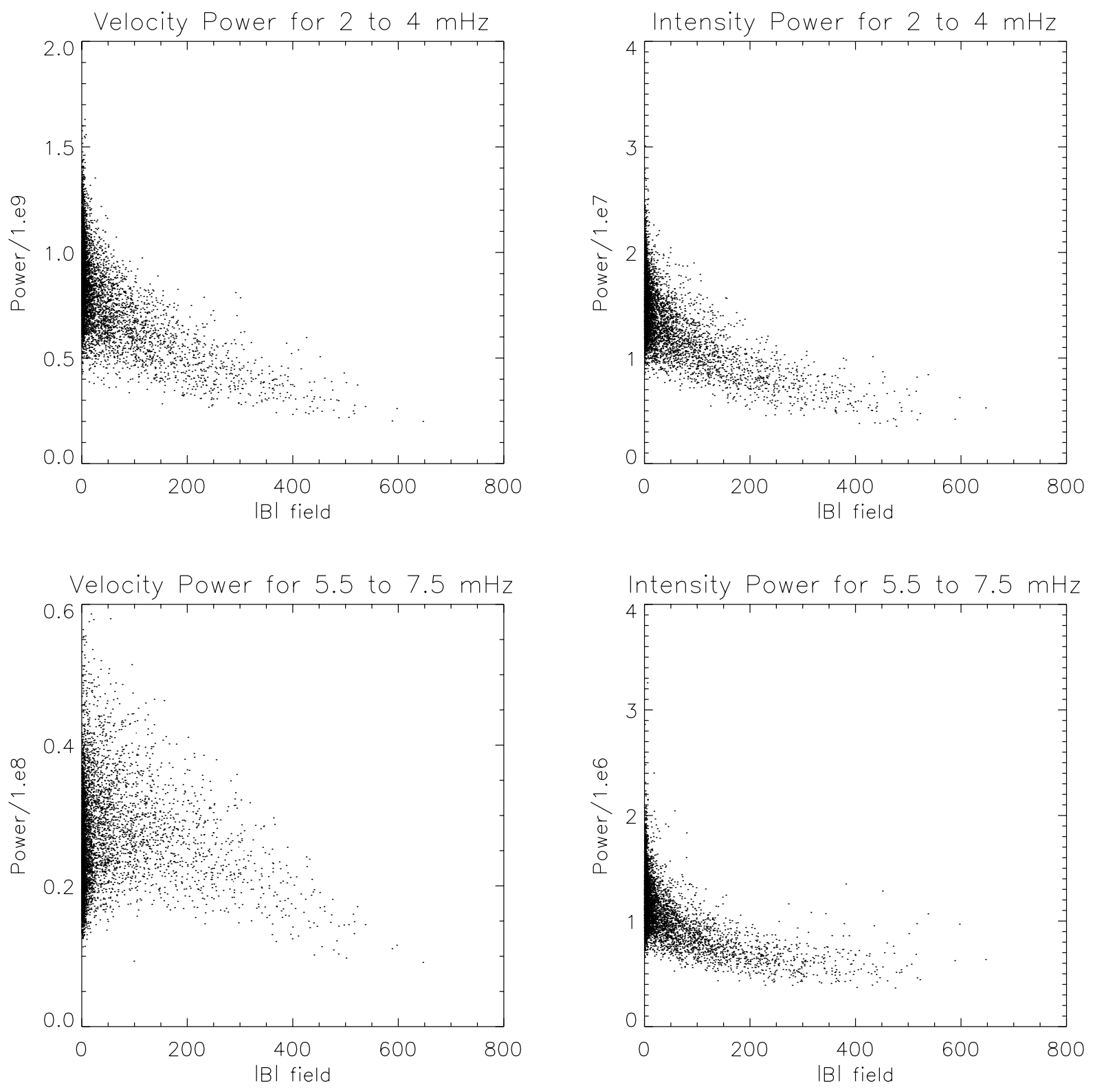

Fig. 4. The Doppler velocity power (first column) and the intensity power (second column) for the "plage" case, in each pixel integrated over the frequency range a) $2 \leq \nu \leq 4 \mathrm{mHz}$ and b) $5.5 \leq \nu \leq 7.5 \mathrm{mHz}$, as a function of the local magnetic field strength.

that both the intensity and the velocity fluctuations reflect changes in the physical conditions at the same height in the atmosphere, we are saying that both the eigenfunctions are affected equally and in the same manner. Radiative transfer effects could cause a breakdown of this assumption. Any comparison of the intensity and velocity eigenfunction must therefore, include the realistic computation of the radiative transfer, including the dynamical effects of the waves. Also, the background power in the intensity and velocity fluctuations could produce different background power levels in the continuum intensity and Doppler velocity power spectra of the plage region.

Before discussing the nature of oscillations in Sunspots and Plage regions, it is necessary to consider the structure of these regions themselves. It is not certain if these magnetic field structures consist of single tubes of magnetic flux or bundles of smaller tubes or whether the magnetic fields expand into a magnetic canopy at the height of formation of spectral lines.

For a flux tube bundle, the effect of tubes on each other and their oscillations is considered beyond the scope of this study. It is difficult to determine observationally the ratio of the velocity amplitudes parallel to and transverse to the magnetic field in sunspots and plages but the general belief is that motions are predominantly vertical near the surface and hence may be aligned with the field. However, modes which have motion dominantly in the horizontal plane cannot be ruled out completely.

If the magnetic region consists of many fragmented thin tube-like structures, surface modes (e.g. 


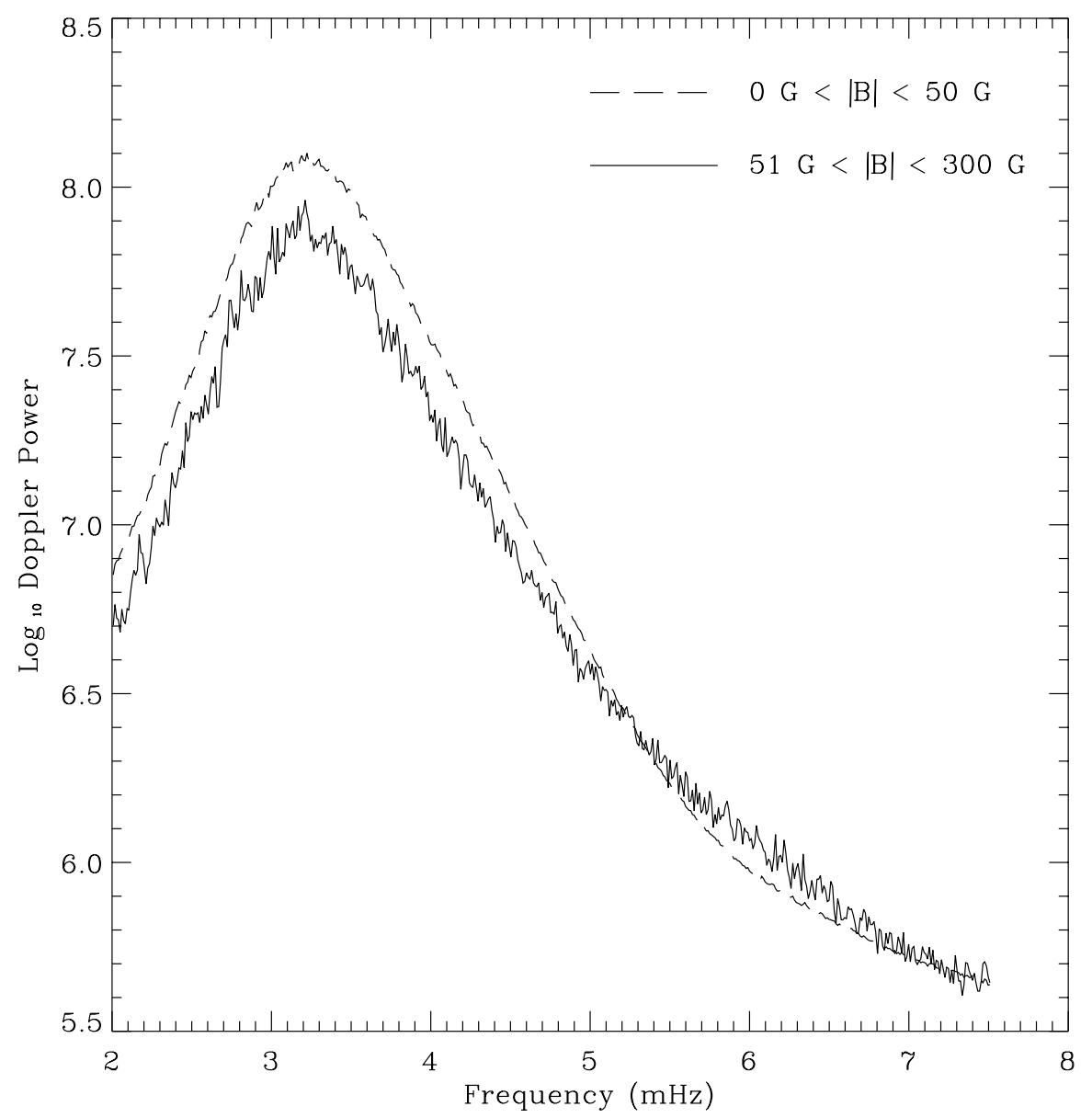

Fig. 5. $\log _{10}$ of the observed Doppler power (for June 6, 1996 data) for two ranges of magnetic field strengths $10 \leq|B| \leq 50 \mathrm{G}$ (dashed line) and $51 \leq|B| \leq 300 \mathrm{G}$ (solid line). Note that for frequencies above $5.3 \mathrm{mHz}$, the greater power belongs to the strong field case whereas below $5.3 \mathrm{mHz}$, the opposite applies.

Roberts 1991) will produce rings of high velocity amplitudes outlining each of these tubes (although one cannot rule out the effect of interaction of tubes). It is possible that there is some contribution of this effect in the highfreq. band for Doppler power maps. However, the fact that the power enhancement is not seen in the continuum intensity data (in the high- $\nu$ band) tempts one to consider incompressible oscillations such as Alfvén waves or transverse kink waves, as the cause for the enhancement in the Doppler velocity data.

Keeping in mind the above-mentioned difficulties with magnetic structure of sunspots and plage regions and the physical background of the intensity and velocity fluctuations, one can only speculate about the physical mechanisms responsible for power suppression and enhancement.

In this study, using MDI observations of Doppler velocity, line-depth, continuum intensity and magnetograms, we have shown that the surface amplitudes of the solar oscillations are suppressed ( $p$-modes) and enhanced (high- $\nu$ oscillations) due to magnetic fields. In general, the magnetic fields seem to influence solar oscillations in the following manners:

1) $p$-mode power is suppressed in the magnetic region and the suppression increases with field strength;

2) high-frequency power is enhanced in and/or around the magnetic region, mainly in pixels with weak and intermediate magnetic field strengths;

3) high-frequency power is still suppressed in the pixels with strong magnetic fields.

The suppression of $p$-mode power is seen in Doppler velocity, line depth and continuum intensity datasets. The maximum suppression appears to be around $3 \mathrm{mHz}$. It has been suggested that the efficiency of $p$-mode excitation by turbulent convection is reduced in the magnetic region (Goldreich \& Keely 1977; Goldreich \& Kumar 1988; Hughes \& Proctor 1989). Another possibility is that the magnetic regions such as sunspots are strong absorbers of p-mode power (Braun et al. 1987, 1988; see also Haber et al. 1999).

Above $5 \mathrm{mHz}$, there is power enhancement in the Doppler velocity and line depth data but not in the continuum intensity. This increased power is seen around the 


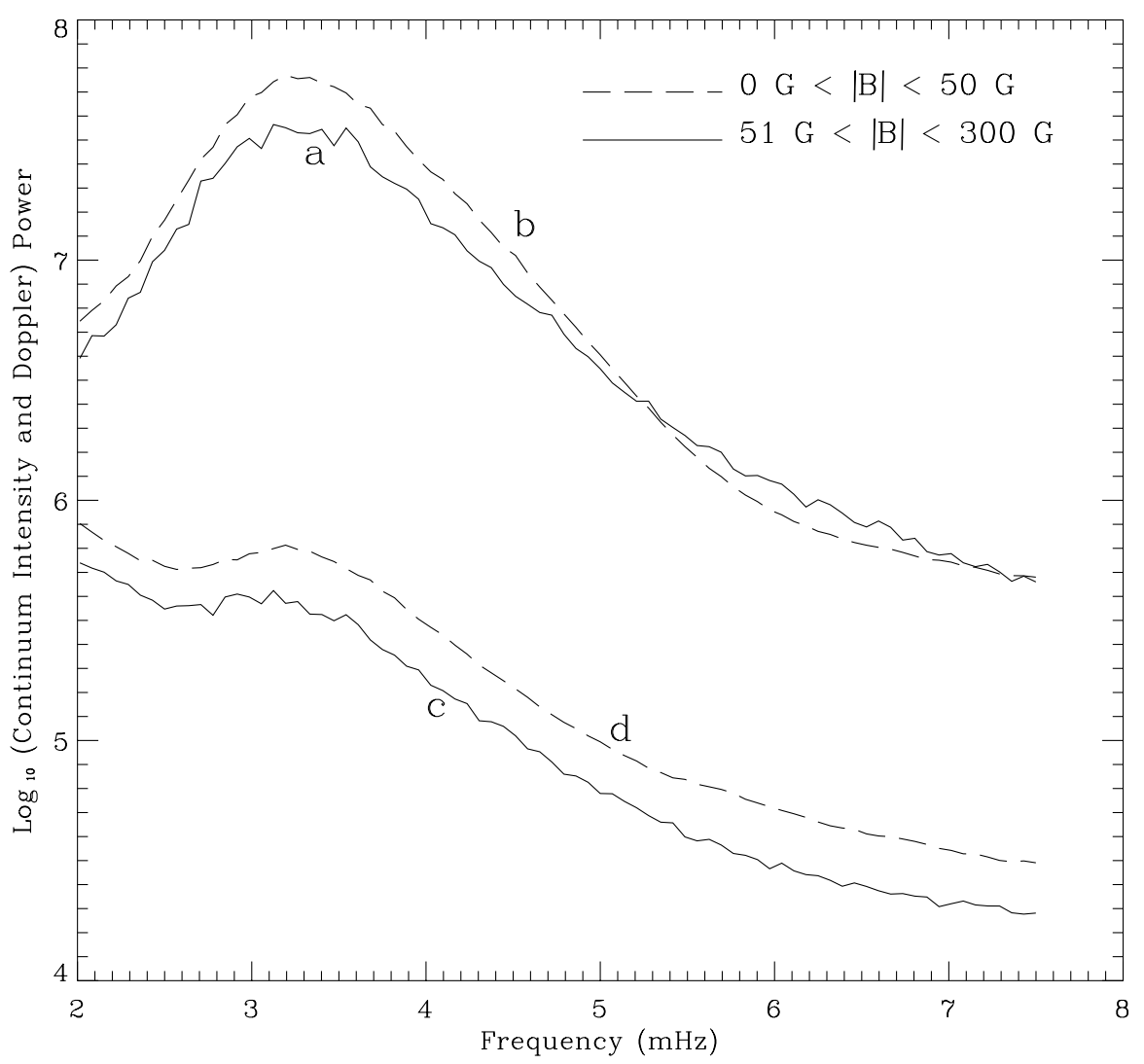

Fig. 6. $\log _{10}$ of the observed Doppler (a and b) and Continuum intensity (c and d) power for Jan. 17, 1997 data for two ranges of magnetic field strengths $10 \leq|B| \leq 50 \mathrm{G}$ (dashed line) and $51 \leq|B| \leq 300 \mathrm{G}$ (solid line). Note from the Doppler power curves $(\mathrm{a}$ and $\mathrm{b}$ ) that for frequencies above $5.3 \mathrm{mHz}$, the greater power belongs to the strong field case whereas below $5.5 \mathrm{mHz}$, the opposite applies. The continuum intensity power for weak field is higher for all frequencies.

strong magnetic region for the sunspot case (see Figs. 2-4 top panel). Keeping in mind that the MDI magnetic field strengths have an uncertainity of $\pm 20 \mathrm{G}$ and that the magnetic regions evolve during the four hours of observation, one has to exercise caution when associating actual magnetic field strength with power suppression and enhancement. However, what is remarkable is that the power enhancement is in the pixels with weak to intermediate magnetic field strengths which are in the immediate surroundings of the strong magnetic field regions.

Knowledge of the photospheric height where the spectral line is formed is vital in order to interpret the completely different behaviour of $p$-mode eigenfunction in the high-frequency band in intensity and velocity data. A paucity of observed evidence for the enhancement in the continuum intensity data is itself insufficient to infer its absence as the enhancement seen in the high-frequency could be from regions with increased acoustic emissivity or scattering (Braun et al. 1992; Brown et al. 1992). This is completely consistent with the lack of observed evidence in the continuum intensity data as the latter samples a different (i.e. deeper) region where the temperature variations induced by the acoustic oscillations may be quite small. Hence, increased acoustic emission in the vicinity of the magnetic region cannot be ruled out, thus leading to the suggestion that the halo-like structure seen in Fig. 2 is acoustic in nature. The line-depth data also gives the information about the temperature variation but in the intermediate photospheric region (between Doppler velocity and continuum intensity) where the waves may no longer be purely isothermal (or adiabatic). One has to be careful with this interpretation because of the opacity variations induced by the temperature variation (see for example, Ruiz Cobo et al. 1997). The line-depth measurements are also liable to contamination from Doppler motion of the spectral line. Thus, line-depth data may show characteristics of both, the continuum intensity data and the Doppler velocity data. On the other hand, if the intensity and velocity perturbations are from more or less the same region of the photosphere then the enhancement of power seen in the Doppler velocity data could be due to the incompressible mode of oscillations such as transverse kink waves.

In conclusion, the physical interpretation for the behaviour of high frequency oscillations $(\nu>4.5 \mathrm{mHz})$ in the presence of a magnetic field is not straightforward. The knowledge of height of spectral-line formation, the propagating or standing nature of the observed waves at this height and the phase difference between the velocity and temperature oscillations are crucial points in untangling the behaviour. Until these points are sorted out, a 
definite conclusion about the mechanism responsible for the amplitude enhancement of high-frequency oscillations around a magnetic region, will be difficult to reach. In the mean time, it is the opinion of the authors that the power enhancement is due to increased acoutic emission.

Acknowledgements. MDI (SOHO) is a project of international cooperation between ESA and NASA. This research was supported by the SOI-MDI NASA Investigation at JILA, University of Colorado, Boulder and by PPARC, UK.

\section{References}

Bogart, R. S., Sá, L. A. D., Duvall, T. L., Jr., et al. 1995, Proc. of Fourth SOHO Workshop, ESA SP-376, 2, 147

Bogdan, T. J., Brown, T. M., Lites, B. W., \& Thomas, J. H. 1993, ApJ, 406, 723

Braun, D. C. 1995, ApJ, 451, 859

Braun, D. C., Duvall Jr., T. L., \& LaBonte, B. J. 1987, ApJ, 319, L27

Braun, D. C., Duvall Jr., T. L., \& LaBonte, B. J. 1988, ApJ, 335,1015

Braun, D. C., Duvall, Jr., T. L., LaBonte, B. J., et al. 1992, ApJ, 391, L113

Braun, D. C., Lindsey, C., Fan, Y., \& Jefferies, S. M. 1992, ApJ, 392, 739

Brown, T. M., Bogdan, T. J., Lites, B. W., \& Thomas, J. H 1992, ApJ, 394, L65
Brown, T. M. 1994, GONG 94, oral presentation

Cally, P. S. 1995, ApJ, 451, 372

Cally, P. S., \& Bogdan, T. M., 1993, ApJ, 402, 721

Goldreich, P., \& Keeley, D. A. 1977, ApJ, 212, 243

Goldreich, P., \& Kumar, P. 1988, ApJ, 326, 462

Haber, D, Jain, R., \& Zweibel, E. G. 1999, ApJ, 515, 832

Hindman, B. W., Jain, R., \& Zweibel, E. G. 1997, ApJ, 476, 392

Hindman, B. W., \& Brown, T. M. 1998, ApJ, 504, 1029

Hughes, D. W., \& Proctor, M. R. E. 1989, Ann. Rev. Fluid Mech., 20, 187

Jain, R., Hindman, B. W., \& Zweibel, E. G. 1996, ApJ, 464, 476

Jain, R., \& Haber, D. 1997, SPD June 26-July 1, 1997 held in Bozeman, Montana, poster presentation

Lites, B. W., White, O. R., \& Packman, D. 1982, ApJ, 253, 386

Roberts, B. 1991 in Advances in Solar System Magnetohydrodynamics, ed. E. R. Priest \& A. W. Hood (Cambridge University Press)

Ruiz Cobo, B., Rodriguez Hidalgo, I., \& Collados, M. 1997, 488, 462

Scherrer, P. H., et al. 1995, Sol. Phys., 162, Nos. 1-2, 129

Tarbell, T., Peri, M., Frank, Z., Shine, R., \& Title, A. 1988 in Seismology of the Sun and Sun-like stars, ed. E. Rolfe, ESA SP-286, 315

Thomas, J. H., \& Stanchfield II, D. C. H. 2000, ApJ, 537, 1086

Woods, D. T., \& Cram, L. E. 1981, Sol. Phys., 69, 233 